\title{
Erratum to: Correlations Between Record Events in Sequences of Random Variables with a Linear Trend
}

\author{
Gregor Wergen · Jasper Franke · Joachim Krug
}

Published online: 21 October 2011

(C) Springer Science+Business Media, LLC 2011

\section{Erratum to: J Stat Phys (2011) 144:1206-1222 \\ DOI 10.1007/s10955-011-0307-7}

Equations (30), (32), (33), (34), (41) and (42) of our article contain some errors. The correct equations read

$$
\begin{gathered}
l_{N, N-1}(c) \approx 1-\frac{c}{4 a} N^{2}, \\
I(N)=\xi \frac{\Gamma\left(2-\frac{1}{\xi}\right) \Gamma(N+1)}{\Gamma\left(N+3-\frac{1}{\xi}\right)}, \\
l_{N, N-1}(c) \approx 1-\frac{c N^{3}}{2} \frac{\Gamma\left(2-\frac{1}{\xi}\right) \Gamma(N-1)}{\Gamma\left(N+1-\frac{1}{\xi}\right)}, \\
l_{N, N-1}(c) \approx 1-\frac{c}{2} \Gamma\left(2-\frac{1}{\xi}\right) N^{1+\frac{1}{\xi}}, \\
f(x)=\mu x^{-\mu-1},
\end{gathered}
$$

The online version of the original article can be found under doi:10.1007/s10955-011-0307-7.

G. Wergen · J. Franke · J. Krug $(\bowtie)$

Institute for Theoretical Physics, University of Cologne, Cologne, Germany

e-mail: krug@thp.uni-koeln.de

G. Wergen

e-mail: gw @thp.uni-koeln.de

J. Franke

e-mail: jfranke@ @hp.uni-koeln.de 
and

$$
I(N)=\mu \frac{\Gamma\left(2+\frac{1}{\mu}\right) \Gamma(N+1)}{\Gamma\left(N+3+\frac{1}{\mu}\right)} .
$$

The remaining results and conclusions of the article are not affected. 